Canad. Math. Bull. Vol. 21 (2), 1978

\title{
ZEROS OF NONLINEAR MONOTONE OPERATORS IN HILBERT SPACE*
}

\author{
BY \\ R. SCHÖNEBERG
}

1. Introduction. Around 1960, the Russian mathematician Kachurovski [1] introduced the notion of monotone operators in Hilbert spaces: Let $E$ be a Hilbert space and $X \subset E$. An operator $T: X \rightarrow E$ is said to be monotone, iff

$$
\operatorname{Re}\langle T(x)-T(y), x-y\rangle \geq 0 \text { for all } x, y \in X
$$

This class of mappings turned out to be very important in application to Partial Differential Equations and has been studied by a number of authors (e.g. Brézis, Browder, Crandall, de Figueiredo, Dolph, Gossez, Gupta, Hess, Kato, Lions, Martin, Minty, Opial, Pazy, Rockafellar, and Vidossich).

The purpose of the present paper is to establish some general sufficient conditions for the existence of zeros of demicontinuous monotone operators, which are defined on the closure of an open (not necessarily convex) subset of a Hilbert space.

Our investigations are motivated by the following simple fact:

Proposition 1. Let $E$ be a Hilbert space, $U \subset E$ be open and let $T: \operatorname{cl}(U) \rightarrow E$ be a monotone mapping which has a zero in $U$. Then the following conditions are satisfied:

$\left(A_{1}\right)$ There is $x_{0} \in U$ such that $T(x) \neq t\left(x-x_{0}\right)$ for all $x \in \partial U, t<0$.

$\left(A_{2}\right)$ There is $x_{0} \in U$ such that $\operatorname{Re}\left\langle T(x), x-x_{0}\right\rangle \geq 0$ for all $x \in \partial U$.

$\left(A_{3}\right)$ There is $x_{0} \in U$ such that for all $x \in \partial U,\|T(x)\|^{2} \leq \operatorname{Re}\left\langle T(x), T\left(x_{0}\right)\right\rangle$ only if $T(x)=0$.

(Note that $\left(A_{2}\right)$ implies $\left(A_{1}\right)$ ).

We shall show that under mild boundedness assumptions each of the conditions $\left(A_{i}\right)$ imply that $T$ has a zero in $\operatorname{cl}(U)$, if $T$ is demicontinuous.

These results (and its consequences) extend and unify several known ones and are based on a lemma due to Crandall-Pazy [2] and the Browder-Minty invariance of domain theorem for demicontinuous locally strongly monotone mappings.

Received by the editors April 26, 1977.

*This paper is based on part of the author's dissertation research at RWTH Aachen under the guidance of Prof. Dr. J. Reinermann. 
2. Preliminaries. To make this paper self-contained we will first briefly introduce some terminology and notation: Let $E$ be a Hilbert space, $X \subset E$, $T: X \rightarrow E, x \in E$ and $r>0$.

(a) $K_{r}(x)$ denotes the closed ball of radius $r$ with center $x$. We use $\operatorname{cl}(X)$ to denote the closure of $X$ and $\partial X$ to denote the boundary of $X$. " $\rightarrow$ " resp. " $\rightarrow$ " indicates strong resp. weak convergence.

(b) $T$ is said to be nonexpansive iff

$$
\|T(x)-T(y)\| \leq\|x-y\| \text { for all } x, y \in X .
$$

(c) $T$ is said to be pseudo-contractive iff

$$
\operatorname{Re}\langle T(x)-T(y), x-y\rangle \leq\|x-y\|^{2} \text {. for all } x, y \in X .
$$

(d) $T$ is said to be strongly monotone iff there is $c>0$ such that

$$
\operatorname{Re}\langle T(x)-T(y), x-y\rangle \geq c\|x-y\|^{2} \text { for all } x, y \in X .
$$

(e) $T$ is said to be monotone iff

$$
\operatorname{Re}\langle T(x)-T(y), x-y\rangle \geq 0 \text { for all } x, y \in X .
$$

(f) $T$ is said to be demicontinuous iff for every sequence $\left(y_{n}\right)$ in $X, y_{n} \rightarrow y \in X$ implies $T\left(y_{n}\right) \rightarrow T(y)$.

For reference we state the following lemmas. The first is due to Crandall and Pazy and the second to Browder and Minty.

LEMMA 1. Let $E$ be a Hilbert space, $\left(x_{n}\right)$ a bounded sequence in $E$ and $\left(r_{n}\right)$ a strictly decreasing sequence in $(0, \infty)$ such that

$$
\operatorname{Re}\left\langle r_{n} x_{n}-r_{m} x_{m}, x_{n}-x_{m}\right\rangle \leq 0 \text { for all } n, m \in \mathbb{N} \text {. }
$$

Then $\left(x_{n}\right)$ converges strongly to some $x \in E$.

Proof. [2].

Lemma 2. Let $E$ be a Hilbert space, $U \subset E$ be open and let $T: U \rightarrow E$ be demicontinuous and locally strongly monotone. Then $T[U]$ is open.

Proof. [3]

\section{RESULTS.}

THEOREM 1. Let $E$ be a Hilbert space, $U \subset E$ be open and let $T: \operatorname{cl}(U) \rightarrow E$ be demicontinuous and strongly monotone. If $K \subset E$ is connected such that $K \cap T[U] \neq \emptyset$ and $K \cap T[\partial U]=\emptyset$, then $K \subset T[U]$.

Proof. let $s:=I+T$. Since $T$ is strongly monotone, we have $(+) \operatorname{Re}\langle S(x)-$ $S(y), x-y) \geq(1+c)\|x-y\|^{2}$ for all $x, y \in \operatorname{cl}(U)$. Hence $S$ is strongly monotone and demicontinuous, too. Let $D:=S[U]$. Then $D$ is open by Lemma 2 and since $S[\mathrm{cl}(U)]$ is closed (by $(+))$, we see that $\operatorname{cl}(D) \subset S[\operatorname{cl}(U)]$. 
Therefore $\partial D \subset S[\partial U]$ and we may define $g: \operatorname{cl}(D) \rightarrow E$ by $g(x):=S^{-1}(x)$. Then $(+)$ implies that $g$ is a mapping with Lipschitz constant $(1+c)^{-1}$. By Kirszbraun's theorem [4], $g$ may be extended to a mapping $G: E \rightarrow E$ which has Lipschitz constant $(1+c)^{-1}$, too. It is easily seen (using $\partial D \subset S[\partial U]$ ) that $K \cap(I-g)[D] \neq \emptyset$ and $K \cap(I-g)[\partial D]=\emptyset$. Since $K$ is connected and $I-G$ is a homeomorphism of $E$ onto $E$, this implies $K \subset(I-G)[D]=(I-g)[D]$. Hence $K \subset T[U]$, completing the proof of Theorem 1 .

Corollary 1. Let $E$ be a Hilbert space, $U \subset E$ be open and let $T: \operatorname{cl}(U) \rightarrow E$ be demicontinuous and strongly monotone. Then $T$ has a zero in $U$ iff there is $x_{0} \in U$ such that $\left\|T\left(x_{0}\right)\right\| \leq\|T(x)\|$ for all $x \in \partial U$.

Proof. Apply Theorem 1 to $K:=\left\{t T\left(x_{0}\right): t \in[0,1]\right\}$.

An easy but useful consequence of Corollary 1 is

TheOREM 2. Let $E$ be a Hilbert space, $U \subset E$ be open and let $H:[0$, $1] \times \operatorname{cl}(U) \rightarrow$ E satisfy

(1) For fixed $t \in[0,1], H(t, \cdot)$ is demicontinuous and strongly monotone.

(2) $H(t, x)$ is continuous in $t$, uniformly for $x \in \operatorname{cl}(U)$.

(3) $H(t, x) \neq 0$ for all $t \in(0,1), x \in \partial U$.

(4) There is $x_{0} \in U$ with $\left\|H\left(0, x_{0}\right)\right\| \leq\|H(0, x)\|$ for all $x \in \partial U$. Then $H(1, \cdot)$ has a unique zero in $\operatorname{cl}(U)$.

Proof. We may assume that $H(1, x) \neq 0$ for $x \in \partial U$. This implies

$$
\sum:=\inf \{\|H(t, x)\|: t \in[0,1], x \in \partial U\}>0 .
$$

(Otherwise $H\left(t_{k}, x_{k}\right) \rightarrow 0$ for some sequences $\left(t_{k}\right)$ in $[0,1]$ and $\left(x_{k}\right)$ in $\partial U$. We may assume $t_{k} \rightarrow t$. Then by (2) $H\left(t, x_{k}\right) \rightarrow 0$. Since $H(t, \cdot)$ is strongly monotone, we get $x_{k} \rightarrow x \in \partial U$. But $H(t, \cdot)$ is demicontinuous, so that $H(t, x)=0$, a contradiction).

Let

$$
M:=\{t \in[0,1]: H(t, \cdot) \text { has a zero in } \mathrm{U}\}
$$

Since $0 \in M$ (by (4) and Corollary 1), it suffices to show that $M$ is both open and closed in $[0,1]$. To show that $M$ is closed, we need only reproduce the argument which we used to prove that $\Sigma>0$. To prove that $M$ is open, let $t_{0} \in M$ and $y_{0} \in U$ with $H\left(t_{0}, y_{0}\right)=0$. Since $H\left(\cdot, y_{0}\right)$ is continuous (by (2)), there is a neighborhood $N$ of $t_{0}$ in $[0,1]$ such that $t \in N$ implies $\left\|H\left(t, y_{0}\right)\right\|<\Sigma$. By definition of $\Sigma$ we get (observing Corollary 1) $N \subset M$. Therefore $M$ is open in $[0,1]$.

We are now in the position to prove our first result for mappings, which are merely monotone. 
Theorem 3. Let $E$ be a Hilbert space, $U \subset E$ be open and let $T: \operatorname{cl}(U) \rightarrow E$ be a demicontinuous monotone mapping such that $(I-T)[\mathrm{cl}(U)]$ is bounded. Then $T$ has a zero in $\mathrm{cl}(U)$; if $\left(A_{1}\right)$ is fulfilled.

Proof. By replacing $T(x)$ with $T\left(x+x_{0}\right)$ and $U$ by $U-x_{0}$, one may take $x_{0}=0$ in $\left(A_{1}\right)$ (and thus $\left.0 \in U\right)$. If we define

$$
M:=\{x \in \operatorname{cl}(U): T(x)=t x \text { for some } t<0\},
$$

then $M$ is bounded (indeed, if $x \in M$, we get $\|T(x)\| \leq\|T(0)\|$, since $T$ is monotone, and thus $\|x\| \leq\|x-T(x)\|+\|T(0)\|)$. Hence we may assume that $U$ is bounded. For $n \in \mathbb{N}$ let now $T_{n}: \operatorname{cl}(U) \rightarrow E$ be defined by $T_{n}(x):=$ $T(x)+(1 / n) x$. Then $T_{n}$ is a demicontinuous strongly monotone mapping which satisfies $\left(A_{1}\right)$, too. Hence $T_{n}$ has a unique zero $x_{n} \in \operatorname{cl}(U)$ by Theorem 2 (use $\left.H(t, x):=(1-t) x+t T_{n}(x)\right)$. Since $T$ is monotone, we get for $n, m \in \mathbb{N}$

$$
\operatorname{Re}\left\langle\frac{1}{n} x_{n}-\frac{1}{m} x_{m}, x_{n}-x_{m}\right\rangle=-\operatorname{Re}\left\langle T\left(x_{n}\right)-T\left(x_{m}\right), x_{n}-x_{m}\right\rangle \leq 0 .
$$

Therefore $\left(x_{n}\right)$ converges strongly to some $x \in \operatorname{cl}(U)$ by Lemma 1. Since $T\left(x_{n}\right)=-(1 / n) x_{n} \rightarrow 0$, we have $T(x)=0$, completing the proof of Theorem 3 .

Corollary 2. Let $E$ be a Hilbert space, $U \subset E$ be open and let $f: \operatorname{cl}(U) \rightarrow E$ be a demicontinuous pseudo-contractive mapping such that $f[\mathrm{cl}(U)]$ is bounded. Suppose there is $x_{0} \in U$ such that

$$
f(x)-x_{0} \neq t\left(x-x_{0}\right) \quad \text { for all } \quad x \in \partial U, \quad t>1 .
$$

Then $f$ has fixed point in $\operatorname{cl}(U)$.

Proof. Apply Theorem 3 to $T=I-f$.

Corollary 2 generalizes Theorem 3 of Browder [5], where $f$ is assumed to be nonexpansive and $U$ is an open ball, and Theorem 4 of ReinermannSchöneberg [6], where in addition $f$ is assumed to be a $k$-set-contraction (in the sense of the Kuratowski-measure of noncompactness) for some $k \geq 0$.

Corollary 3. Let $E$ be a Hilbert space, $r>0$ and let $f: K_{r}(0) \rightarrow E$ be nonexpansive. Then $f$ has a fixed point in $K_{r}(0)$ iff

$$
f(x) \neq t x \text { for all }\|x\|=r, \quad t>1 .
$$

Proof. If $f$ satisfies $(*)$, then $f$ has a fixed point in $K_{r}(0)$ by Corollary 2 . Conversely, suppose $f$ has a fixed point, say $x_{0} \in K_{r}(0)$, and let $\|x\|=r$ and $t \geq 0$ with $f(x)=t x$. We have to show that $t \leq 1$. Since $f$ is nonexpansive, we get

i.e.,

$$
\left\|t x-x_{0}\right\|^{2}=\left\|f(x)-f\left(x_{0}\right)\right\|^{2} \leqq\left\|x-x_{0}\right\|^{2},
$$

$$
2(1-t) \operatorname{Re}\left\langle x, x_{0}\right\rangle \leq(1-t)(1+t)\|x\|^{2} .
$$


If $t>1$, this implies (using $\operatorname{Re}\left\langle x, x_{0}\right\rangle \leq\|x\|^{2}$ ) $1+t \leq 2$, i.e., $t \leq 1$, a contradiction. Hence $t \leq 1$.

THEOREM 4. Let $E$ be a Hilbert space, $U \subset E$ be open and bounded and let $T: \mathrm{cl}(U) \rightarrow E$ be demicontinuous and monotone. Then $T$ has a zero in $\mathrm{cl}(U)$, if the condition $\left(A_{2}\right)$ or $\left(A_{3}\right)$ is satisfied.

Proof. We may assume $T(x) \neq 0$ for all $x \in \partial U$. Let $S:=I+T$. Since $T$ is monotone, we have

$(++)$

$$
\operatorname{Re}\langle S(x)-S(y), x-y\rangle \geq\|x-y\|^{2} \text { for all } x, y \in \operatorname{cl}(U) .
$$

If $g$ and $D$ are defined as in the proof of Theorem 1, then $(++)$ implies that $g$ is nonexpansive. Let $x_{0} \in U$ be the point specified in the statement of $\left(A_{2}\right)$ resp. $\left(A_{3}\right)$ and let $\bar{x}_{0}:=S\left(x_{0}\right)$. In view of Corollary 2 it suffices to show that the assumption $g(\bar{x})-\bar{x}_{0}=t\left(\bar{x}-\bar{x}_{0}\right)$ for some $\bar{x} \in \partial D, t>1$ leads to a contradiction. Let $x:=g(\bar{x})$. Then $x \in \partial U$ (recall $\partial D \subset S[\partial U]$ ) and $\bar{x}=x+T(x)$. Therefore $(t-1) T\left(x_{0}\right)=(t-1)\left(x-x_{0}\right)+t T(x) .\left(A_{2}\right)$ : The monotonicity of $T$ implies

i.e.,

$$
\begin{aligned}
0 & \leq \operatorname{Re}\left\langle T(x)-T\left(x_{0}\right), x-x_{0}\right\rangle \\
& =\operatorname{Re}\left\langle T(x)-\left(x-x_{0}\right)-\frac{t}{t-1} T(x), x-x_{0}\right\rangle,
\end{aligned}
$$

$$
\operatorname{Re}\left\langle T(x), x-x_{0}\right\rangle \leq-(t-1)\left\|x-x_{0}\right\|^{2}<0,
$$

a contradiction.

$\left(A_{3}\right)$ : Again the monotonicity of $T$ implies, using $t-1>0$,

hence

$$
\begin{aligned}
0 & \leq(t-1) \operatorname{Re}\left\langle T(x)-T\left(x_{0}\right), x-x_{0}\right\rangle \\
& =\operatorname{Re}\left\langle T(x)-T\left(x_{0}\right),(t-1)\left(x-x_{0}\right)\right\rangle \\
& =\operatorname{Re}\left\langle T(x)-T\left(x_{0}\right),(t-1) T\left(x_{0}\right)-t T(x)\right\rangle,
\end{aligned}
$$

i.e.,

$$
\begin{aligned}
\operatorname{Re}\left\langle T(x)-T\left(x_{0}\right), T\left(x_{0}\right)\right\rangle & \leq-t\left\|T(x)-T\left(x_{0}\right)\right\|^{2} \\
& \leq-\left\|T(x)-T\left(x_{0}\right)\right\|^{2},
\end{aligned}
$$

$$
\operatorname{Re}\left\langle T(x)-T\left(x_{0}\right), T\left(x_{0}\right)\right\rangle+\left\|T(x)-T\left(x_{0}\right)\right\|^{2} \leq 0 .
$$

But this is equivalent to $\|T(x)\|^{2} \leq \operatorname{Re}\left\langle T(x), T\left(x_{0}\right)\right\rangle$. Hence $T(x)=0$ by $\left(A_{3}\right)$, contradicting our assumption.

Corollary 4. Let $E$ be a Hilbert space, $U \subset E$ be open and bounded and let $T: \operatorname{cl}(U) \rightarrow E$ be a demicontinuous and monotone mapping. Suppose there is $x_{0} \in U$ such that $\left\|T\left(x_{0}\right)\right\|<\|T(x)\|$ for all $x \in \partial U$. Then $T$ has a zero in $U$.

Proof. $\left(A_{3}\right)$ is fulfilled. 
Corollary 4 is a sort of a minimum principle for monotone mappings. It has been proved by Kirk-Schöneberg [7] (for accretive mappings in general Banach spaces) under the additional assumptions that $T$ is continuous and $\operatorname{cl}(U)$ has the fixed-point property with respect to nonexpansive self-mappings.

Corollary 5. Let $E$ be a Hilbert space, $U \subset E$ be an open bounded symmetric neighborhood of the origin and let $T: \mathrm{cl}(U) \rightarrow E$ be demicontinuous and monotone. If $T(x)=-T(-x)$ for all $x \in \partial U$, then $T$ has a zero in $\mathrm{cl}(U)$.

Proof. Since $T$ is monotone, we get for $x \in \partial U$

and

$$
\operatorname{Re}\langle T(x)-T(0), x\rangle \geq 0
$$

Hence

$$
\operatorname{Re}\langle T(0)-T(-x), x\rangle \geq 0
$$

$$
2 \operatorname{Re}\langle T(x), x\rangle \geq 0
$$

Therefore $\left(A_{2}\right)$ is fulfilled.

Corollary 5 extends Theorem 5 of Browder [5], where $U$ is an open ball and $T$ is assumed to be defined on all of $E$. It generalizes Theorem 7 of Reinermann-Stallbohm [8], where $T=I-f$ with $f$ nonexpansive, and Theorem 5 of Reinermann-Schöneberg [6], where $T$ is in addition assumed to be lipschitzian.

4. Concluding remarks. For clarity of exposition, we have restricted ourselves to the investigation of monotone operators in Hilbert spaces. Some of our results however, hold also for the class of accretive mappings in reflexive $(\pi)_{1}$-spaces that admit a weakly continuous duality mapping. This depends mainly on the fact that a lemma of Crandall-Pazy type is true in reflexive Banach spaces having a weakly continuous duality mapping (see Krauthausen et al. [9]). The reader, who is interested in these slight generalizations, can easily carry through the proofs using the ideas of this paper and the results of [9] and [10].

\section{REFERENCES}

1. R. I. Kachurovski, On monotone operators and convex functionals, Uspehi Mat. Nauk 15, 213-215 (1960).

2. M. Crandall and A. Pazy, Semigroups of nonlinear contractions and dissipative sets, J. Functional Analysis 3, 376-418 (1969).

3. F. E. Browder, Remarks on non-linear functional equations III, Illinois J. Math. 9, 617-622 (1965).

4. I. J. Schoenberg, On a theorem of Kirszbraun and Valentine, Amer. Math. Monthly 60, 620-622 (1953).

5. F. E. Browder, Fixed point theorems for noncompact mappings in Hilbert space, Proc. Nat. Acad. Sci. U.S.A. 53, 1272-1276 (1965).

6. J. Reinermann and R. Schöneberg, Some results in fixed point theory for nonexpansive and 
pseudo-contractive mappings in Hilbert space, Proceedings of a Seminar on Fixed Point Theory and its Applications, Dalhousie University, Halifax, N.S., June 9-12 (1975), Academic Press.

7. W. A. Kirk and R. Schöneberg, Some results on pseudo-contractive mappings, Pacific J. Math., 71, 89-100 (1977).

8. J. Reinermann and V. Stallbohm, Fixed point theorems for compact and nonexpansive mappings on starshaped domains, Math. Balkanica 4.95, 511-516 (1974).

9. Cl. Krauthausen, G. Müller, J. Reinermann and R. Schöneberg, New fixed point theorems for compact and nonexpansive mappings and applications to Hammerstein equations, Sonderforschungsbereich 72, Universität Bonn, preprint no. 92, 108 p., 1976.

10. F. E. Browder and D. G. de Figueiredo, J-monotone nonlinear operators in Banach spaces, Indag. Math. 28, 412-420 (1966).

\section{Lehrstuhl C FÜr MATH., RWTH AACHEN, 5100 AACHEN \\ Fed. Rep. of Germany}

